\title{
Perfil del alumno de la Universidad Tecnológica del Norte de Aguascalientes
}

\section{Student profile of the Universidad Tecnológica del Norte de Aguascalientes}

\author{
DELGADO-RUIZ-ESPARZA, Virginia†* \& GARCÍA-RODRÍGUEZ, Juan Carlos \\ Universidad Tecnológica del Norte de Aguascalientes, Coordinación de Tutorías. Rincón Station, Av. University No. 1001, \\ 20400 Rincón de Romos, Ags.
}

ID $1^{\text {er }}$ Autor: Virginia, Delgado-Ruiz-Esparza / ORC ID: 0000-0002-9644-6511, Researcher ID Thomson: Y-4537-2018

ID $1^{\text {er }}$ Coautor: Juan Carlos, García-Rodríguez / ORC ID: 0000-0002-3602-7809, Researcher ID Thomson: J-8291-2017

\begin{abstract}
Resumen
Para la atención y seguimiento al alumno es importante contar con información que permita llevar a cabo acciones con oportunidad en su trayectoria educativa, con miras a la permanencia y conclusión de su formación profesional. A su vez, que la tarea de seguimiento por parte del tutor esté orientada con base a información del alumno respecto de sus condiciones académicas, socioeconómicas y personales que marcan la pauta de la función tutorial. Derivado de lo anterior, el objetivo es caracterizar al alumno de la Universidad Tecnológica del Norte de Aguascalientes, identificando sus condiciones de vulnerabilidad que deriven en un perfil del alumno, a través del cuestionario inicial del alumno de nuevo ingreso del periodo Septiembre-Diciembre 2018 del nivel de TSU. El instrumento de diagnóstico del alumno, estructurado en tres aspectos, permite identificar condiciones de vulnerabilidad de tipo académico, socioeconómico y/o psicológico, condiciones que detonan parte importante de la función tutorial. Contar con un perfil representa un insumo para la elaboración del Plan de Acción Tutorial por parte de los tutores e incide en las líneas de acción en el Programa Institucional de Tutorías.
\end{abstract}

\section{Tutoría, Perfil, Vulnerabilidad}

\begin{abstract}
For the attention and follow-up of the student it is important to have information that allows carrying out actions with opportunity in their educational trajectory, with a view to the permanence and conclusion of their professional training. In turn, that the follow-up task by the tutor is oriented based on information from the student regarding their academic, socioeconomic and personal conditions that set the guideline for the tutorial function. Derived from the above, the objective is to characterize the student of the Technological University of the North of Aguascalientes, identifying their conditions of vulnerability that derive in a profile of the student, through the initial questionnaire of the newly admitted student of the September-December 2018 period of the TSU level. The student's diagnostic instrument, structured in three aspects, allows to identify conditions of vulnerability of academic, socioeconomic and / or psychological type, conditions that trigger an important part of the tutorial function. Having a profile represents an input for the preparation of the Tutorial Action Plan by the tutors and affects the lines of action in the Institutional Tutoring Program.
\end{abstract}

Tutoring, Profile, Vulnerability

Citación: DELGADO-RUIZ-ESPARZA, Virginia \& GARCÍA-RODRÍGUEZ, Juan Carlos. Perfil del alumno de la Universidad Tecnológica del Norte de Aguascalientes. Revista de Educación Técnica. 2019. 3-10: 25-31.

\footnotetext{
*Correspondencia al Autor (Correo Electrónico: virginia.delgado@utna.edu.mx)

$\dagger$ Investigador contribuyendo como primer autor.
} 


\section{Introducción}

El alumno es el actor central de las Instituciones de Educación Superior (IES), en este sentido es de gran relevancia contar con una estrategia académica como la Tutoría que brinde acompañamiento al alumno en lo individual y/o grupal, a través de la función tutorial con el propósito de incidir en la formación integral del alumno, en su permanencia y egreso. La Tutoría en el Subsistema de Universidades Tecnológicas y de acuerdo con lo especificado en Modelo Nacional de Tutorías "la tutoría constituye una estrategia de apoyo y asesoría para los estudiantes en su proceso de adaptación, desarrollo y formación profesional; además, tiene la finalidad de potenciar sus capacidades y brindar herramientas que ayuden a superar las dificultades que se les presenten a lo largo del proceso educativo.

De manera paralela, contribuye al aprovechamiento académico y mejora de la eficiencia terminal" (CGUTyP, 2017). El Tutor es un profesor, en la mayoría de los casos, de tiempo completo, quien realiza tareas de acompañamiento y seguimiento al alumno, a quien le proporciona información, asesoría y en su caso, lo canaliza cuando el alumno presenta condiciones vulnerabilidad, en los ámbitos académico, socioeconómico y personal (UTNA, 2019). El tutor, como parte de sus funciones, canaliza al alumno a alguna de las áreas de servicio que le permita compensar una situación en los ámbitos citados.

Considérese como condiciones de vulnerabilidad de los alumnos a aquellas "Situaciones que presentan estudiantes por distintas circunstancias de vida que les ponen en riesgo de abandono escolar o bajo desempeño académico. Estas circunstancias pueden derivarse de situaciones académicas, personales o socioeconómicas" (CGUTyP, 2017). Para la atención y seguimiento del alumno es importante contar con información que permita llevar a cabo acciones concretas y con oportunidad durante su trayectoria educativa, ésto con miras a la permanencia y conclusión de su formación profesional. A su vez, la tarea de seguimiento por parte del tutor debe estar orientada con base a información del alumno respecto de sus condiciones académicas, socioeconómicas y personales que marcan la pauta de la función tutorial.
Contar con información específica del alumno sitúa al tutor en una posición que permite trabajar de manera preventiva, con elementos que determinar condiciones concretas de vulnerabilidad del alumno. La importancia de contar con un perfil de la población estudiantil radica en que con esa información se está en condiciones de brindar una atención en congruencia con sus necesidades. Por otra parte, es importante debilitar estigmas respecto de la población atendida por su contexto social, así como es necesario actualizar características de la población estudiantil de una sociedad cada vez más compleja y cambiante.

Derivado de lo anterior, el objetivo es caracterizar al alumno de la Universidad Tecnológica del Norte de Aguascalientes, identificando sus condiciones de vulnerabilidad que deriven en un perfil del alumno, a través del cuestionario inicial del alumno de nuevo ingreso del periodo Septiembre-Diciembre 2018 del nivel de TSU.

Cabe señalar que parte de las acciones dentro del Programa de Tutoría con alumnos de nuevo ingreso es la aplicación del cuestionario inicial que integra información del alumno. Se trata de un instrumento en formato digital. El instrumento de diagnóstico del alumno, el cual está estructurado, además de los datos generales, en tres aspectos, permite identificar condiciones de vulnerabilidad de tipo académico, socioeconómico y/o psicológico, condiciones que detonan parte importante de la función tutorial. Contar con un perfil representa además, contar con un insumo para la elaboración del Plan de Acción Tutorial por parte de los tutores, así como también incide en las líneas de acción del Programa Institucional de Tutorías.

El cuestionario del cual se configura el perfil de los alumnos de nuevo ingreso a la UTNA en el periodo 2018-2019, es de 970 casos, de los cuales 427 son mujeres y 543 son hombres, y no necesariamente representa la matrícula total de ese periodo.

El cuestionario, además de los datos generales, está estructurado en los tres apartados citados y cuenta con 65 preguntas que pretenden conocer sobre el nivel de vulnerabilidad de los alumnos en esos ámbitos. 


\section{Resultados del cuestionario}

De los resultados que arrojó el cuestionario se presentan en primer lugar los datos generales, y en seguida, los referentes a los ámbitos socioeconómico, personal y académico. Estos resultados configuran un perfil del estudiante de la Universidad Tecnológica del Norte de Aguascalientes (UTNA).

\section{Datos generales}

Este perfil del alumno de la UTNA se construye con base a un $44 \%$ de mujeres y un $56 \%$ de hombres: de esta población el $84 \%$ es originaria del Estado de Aguascalientes y el 16\% del estado de Zacatecas. Contribuyen en ese $84 \%$ alumnos originarios de los municipios de Rincón de Romos, Pabellón de Arteaga, San Francisco de los Romo y Asientos, principalmente.

Por su parte, de Zacatecas provienen fundamentalmente de los municipios de Ojocaliente, Loreto, Luis Moya y Ciudad Cuauhtémoc.

Todos ellos se han inscrito en alguno de los Programas Educativos (PE) que oferta la UTNA, con acentuada presencia en los PE de MI, ACH, CON, MTAUT y PIM; un $48 \%$ estudia un PE del ámbito económico administrativo, considerándose en este ámbito los estudiantes de Tecnologías de la Información; y un 52\% de los alumnos estudia en un PE del área de las ingenierías.

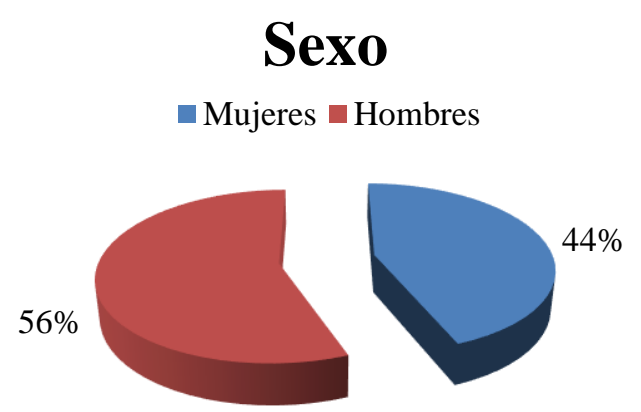

Gráfico 1 Sexo

Fuente: elaboración propia

\section{Estado de procedencia del alumno}

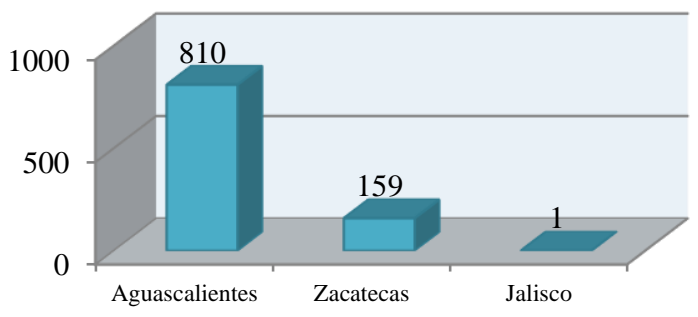

Gráfico 2 Estado de procedencia del alumno Fuente: elaboración propia

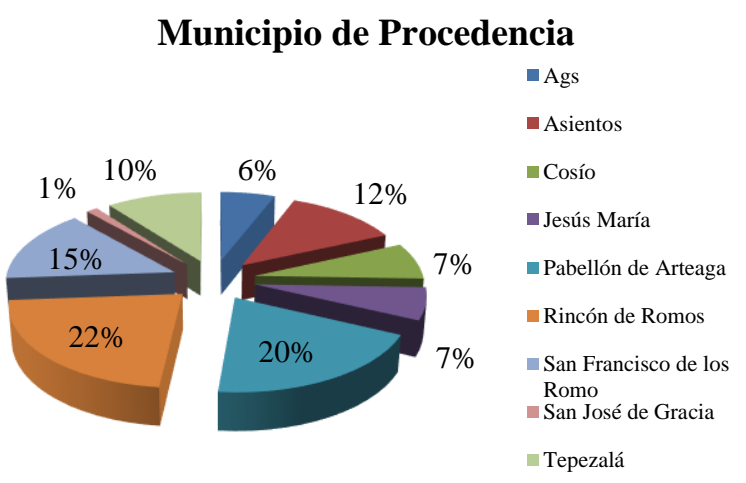

Gráfico 3 Municipio de procedencia

Fuente: elaboración propia

\section{Ámbito socioeconómico}

Los alumnos viven con su padres en el $95 \%$ de los casos y solamente el $4 \%$ vive con sus abuelos, algún hermano, tío o tutor; el resto vive con su pareja, amigos o solo.

Los alumnos pertenecen a una familia conformada, en promedio, por 5 integrantes; sin embargo, hay alumnos que viven solos y en otro escenario comparten su vivienda con hasta 15 miembros de la familia. No obstante, el $70 \%$ de los alumnos tiene de 1 a 3 hermanos; el $23 \%$ tiene de 4 a 6 hermanos; el $4 \%$ tiene 7 o más hermanos, el resto no tiene. El $65 \%$ de los hermanos de los alumnos estudia y el $23 \%$ trabaja como empleado.

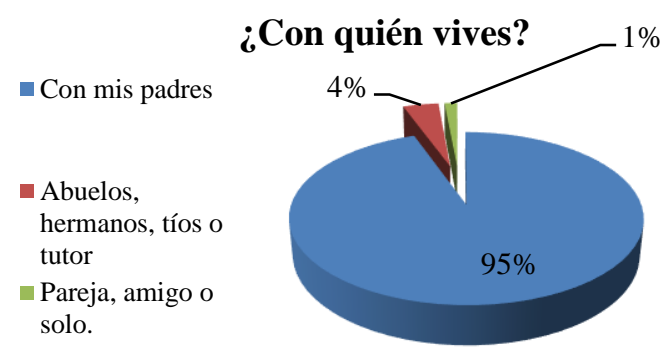

Gráfico 4 ¿Con quién vives?

Fuente: elaboración propia 
Los alumnos de esta universidad tienen como actividad principal estudiar, debido a que el $76 \%$ no trabaja, únicamente el $24 \%$ de los alumnos desempeña una actividad laboral. De los alumnos que trabajan el $51 \%$ trabaja de 5 a 10 horas semanales, el $27 \%$ de 10 a 20 horas a la semana y el $21 \%$ trabaja 20 horas o más. Asimismo, cabe señalar que el ingreso mensual que perciben los alumnos es de 100 a 800 pesos en un $76 \%$; y un $23 \%$ de los estudiantes que trabaja percibe ingresos de 801 a 3,000 pesos y más. Con todo, el $94 \%$ de los alumnos indican que dependen sus padres; el 6\% restante depende de sí mismo, un familiar o amigo.

En el $47 \%$ de los alumnos que son hijos de padres que trabajan, perciben ingresos mensuales de hasta 2,699 pesos; mientras que en el $44 \%$ de los casos, el ingreso familiar mensual es de 2,700, 6,799 pesos; y solamente, el 9\% restante tiene ingresos familiares de 6,800 pesos y más. La ocupación preponderante del padre de familia es en el $51 \%$ como empleado: a su vez, el $25 \%$ de los padres de familia se dedican a las labores del campo, como agricultores o jornaleros. Asimismo, el $7 \%$ de los padres de familia realiza actividades comerciales o de servicios y el $3 \%$ se dedica a la construcción. Por su cuenta, las madres de familia se ocupan en las labores del hogar en un $75 \%$, un $19 \%$ de ellas trabaja como empleada y apenas un $4 \%$ se dedica a la actividad comercial o de servicios.

\section{Ingreso familiar mensual}

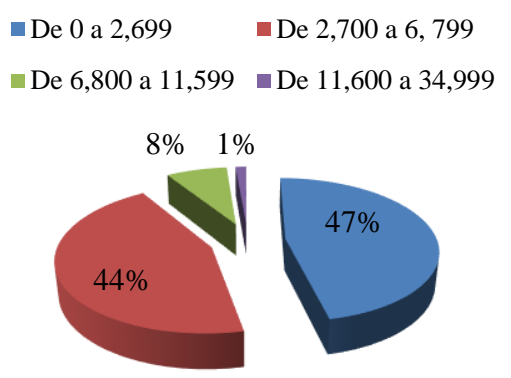

Gráfico 5 Ingreso familiar mensual Fuente: elaboración propia

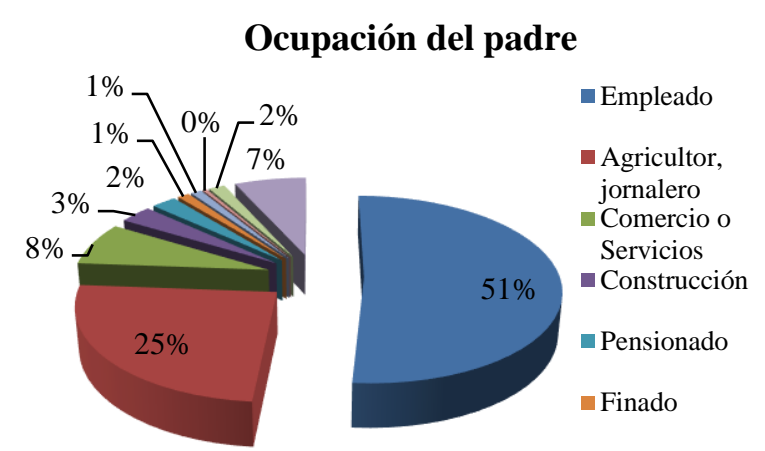

Gráfico 6 Ocupación del padre

Fuente: elaboración propia

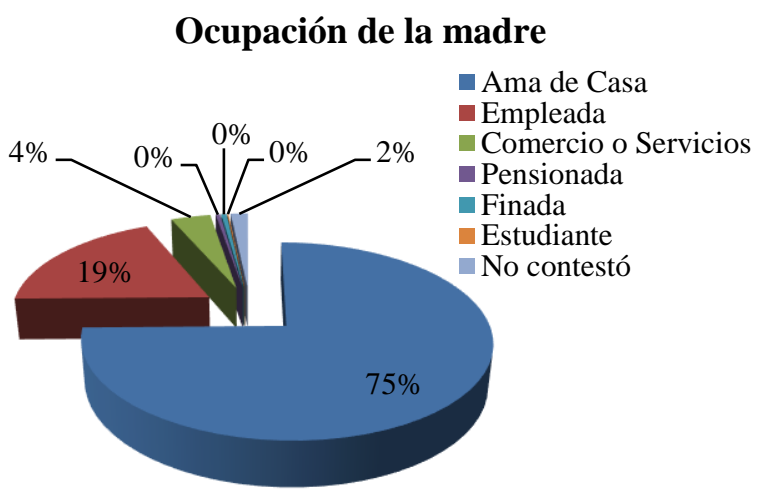

Gráfico 7 Ocupación de la madre

Fuente: elaboración propia

Respecto de la vivienda que habitan el $79 \%$ de los alumnos cuenta con vivienda propia; el $9 \%$ ocupa una vivienda prestada y en el $9 \%$ de los alumnos la vivienda que ocupa es en calidad de rentada.

\section{Ámbito personal}

Respecto al padecimiento de alguna enfermedad, el $91 \%$ de los alumnos descarta tener problemas de salud, mientras que solo el 9\% afirma tener algún problema de salud; los problemas de salud son el $67 \%$ alergias, el $6 \%$ crónico degenerativas y el resto son otras enfermedades o no contestaron.

Respecto de un grupo de aspectos específicos de salud, el $48 \%$ indicó no presentar problemas. 


\section{Enfermedad que padece}

$\begin{array}{ll}\square \text { Alergias } & \text { Crónico degenerativas } \\ \square \text { Otras } & \text { No contestó }\end{array}$

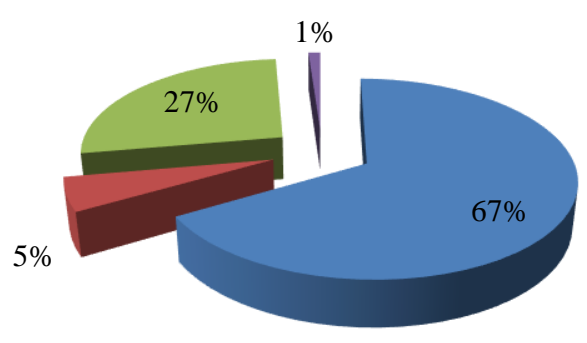

Gráfico 8 Enfermedad que padece

Fuente: elaboración propia

A su vez, un $25 \%$ de los alumnos indicó tener problemas visuales; el $8 \%$ dijo tener problemas de salud relacionados con su dentadura y un porcentaje similar, $8 \%$, externó presentar problemas de obesidad y el resto tiene otras afecciones. Por su parte, el $70 \%$ de los alumnos indicó no presentar alguna discapacidad, en cambio, destaca el $24 \%$ de alumnos que señalaron contar con una discapacidad de tipo visual.

\section{Aspectos que presenta el alumno}

- Problemas visuales

- Problemas de dentadura

n Obesidad

- Falta de energía

- Manchas en la piel 13

- Delgadez extrema

Problemas auditivos

$\square$ Otra

Ninguna

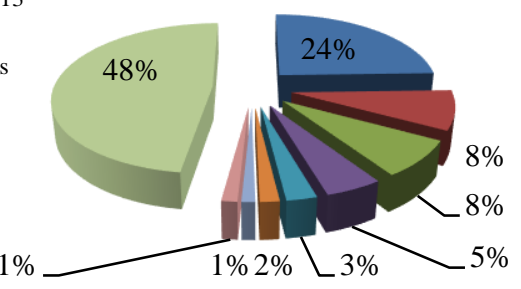

Gráfico 9 Aspectos que presenta el alumno

Fuente: elaboración propia

Del total de la población, el 19\% manifestó haber recibido atención psicológica; no obstante, el $13 \%$ de los estudiantes indicó haber sido diagnosticado en un aspecto psicológico; principalmente trastorno de ansiedad, depresivo y problemas de aprendizaje. Asimismo, en cuanto a factores de convivencia únicamente el 5\% dijo haber experimentado bullying o acasos escolar y exclusión o aislamiento.
Con relación al consumo de sustancias permitidas el $67 \%$ de los alumnos indicó no hacer uso de ellas, si en cambio el $23 \%$ de los alumnos manifestó consumir alcohol, el 9\% restante, hace uso de bebidas energéticas, tabaco y otras sustancias. Respecto de las sustancias no permitidas, los alumnos mencionaron el consumo de la marihuana y otras sustancias en apenas el $1 \%$ de los estudiantes.

¿Consumes alguna sustancia permitida?

Ninguna $\square$ Otra $\square$ Alcohol $\square$ Bebidas energéticas $\square$ Tabaco

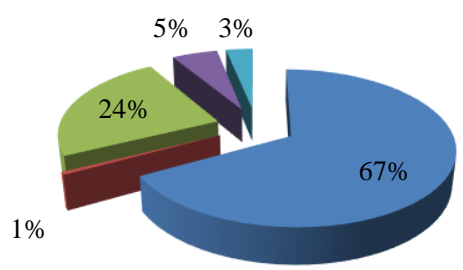

Gráfico 10 ¿Consumes alguna sustancia permitida? Fuente: elaboración propia

Por otra parte, el $3 \%$ de los alumnos manifestó ser padre o madre de familia, además, el $1 \%$ de los alumnos externó estar en ese momento en espera de bebe.

\section{Ámbito académico}

Los alumnos que ingresan a la Universidad proceden de los diferentes subsistemas de educación media superior; el 35\% de los alumnos provienen del Colegio de Estudios Científicos y Tecnológicos de los Estados (CECYTE); en similar proporción, $14 \%$ de los alumnos, realizaron sus estudios en Bachilleratos generales y $14 \%$ hicieron estudios previos en el Centros de Bachillerato Tecnológico Agropecuario (CBTA); a su vez, $8 \%$ de los alumnos egresaron del Centro de Bachillerato Tecnológico y de Servicios (CBTIS) y mismo porcentaje, $8 \%$, realizaron su educación de nivel medio superior en el Telebachillerato comunitario. En menor porcentaje, egresados del Colegio Nacional de Educación profesional Técnica (CONALEP), un $6 \%$ de los alumnos ingresó a realizar sus estudios de nivel superior a esta universidad. El resto de los alumnos, en menor porcentaje, provienen, entre otros de los Centros de Educación Media a Distancia (CEMSAD) y del Colegio de Bachilleres del Estado de Zacatecas (COBAEZ). 
El mayor número de alumnos, 34\% de ellos, presentan evaluaciones con un promedio de 8 a 8.5; a su vez, el $31 \%$ de los alumnos tienen un promedio de 7.1 a 7.9 mientras que en el $27 \%$ de los alumnos registran promedios de 8.6 a 9.4. En los extremos, tenemos un 3\% de alumnos con promedio de 7 o menos y un 5\% con un promedio igual o mayor a $9.5 \%$.

\section{Promedio}
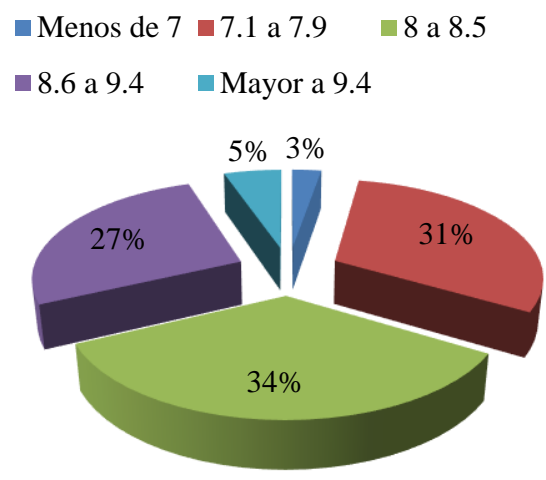

Gráfico 11 Promedio

Fuente: elaboración propia

Aunado al nivel de promedio que presentan los alumnos, el 79\% de ellos dijo haber ingresado a una carrera que era su primera opción, no obstante el $21 \%$ de los alumnos manifestaron haber ingresado a una carrera que no era su primera opción.

\section{¿Tu carrera es tu primera opción?}

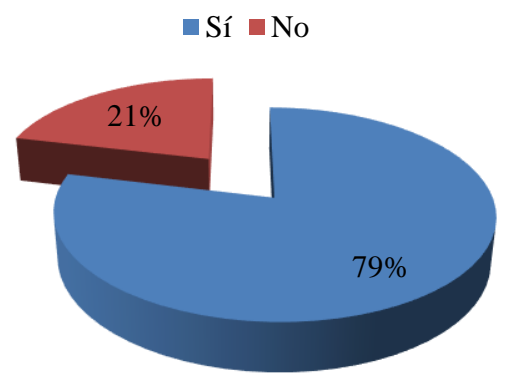

Gráfico 12 ¿Tu carrera es tu primera opción?

Fuente: elaboración propia

Asimismo, el $73 \%$ de los alumnos afirmó contar con una computadora para realizar sus actividades escolares, y por otra parte el $27 \%$ de ellos indicaron no poseer esta herramienta de estudios. Además, el 57\% indicó que utiliza o consulta libros para estudiar y el $43 \%$ externó que los libros no representan un insumo en su actividad académica.

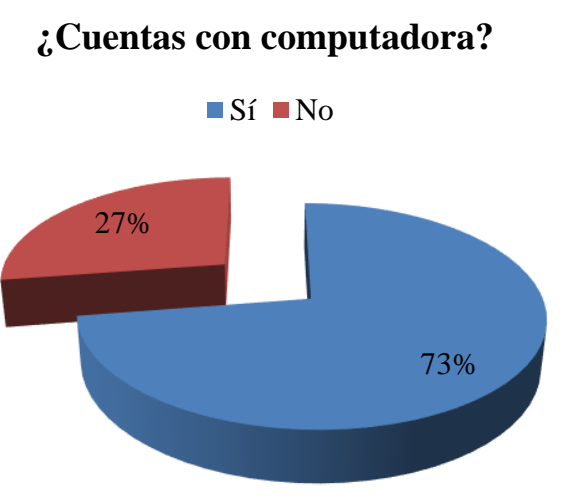

Gráfico 13 ¿Cuentas con computadora? Fuente: elaboración propia

\section{¿Utilizas libros para estudiar?}

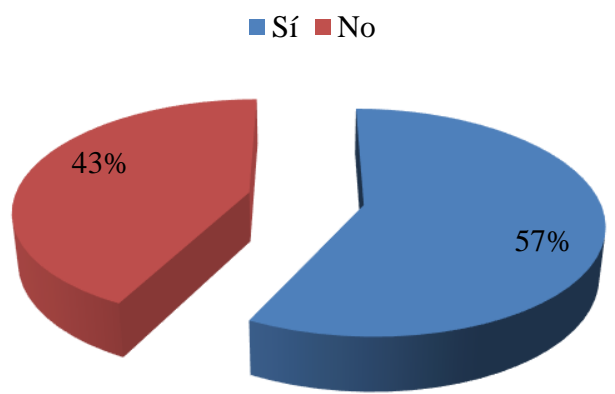

Gráfico 14 ¿Utilizas libros para estudiar? Fuente: elaboración propia

\section{Conclusiones}

Con relación al perfil del alumno de la UTNA, habría que señalar que la edad promedio es de 18 años y que la cantidad de mujeres y hombres está en porcentajes similares, aunque por encima el número de hombres respecto del número de mujeres; fundamentalmente son originarios de los municipios de Rincón de Romos, Pabellón de Arteaga, San Francisco de los Romo y Asientos del Estado de Aguascalientes, quienes alternan con una minoría de alumnos originarios de municipios del sur de Zacatecas. Asimismo, la población estudiantil ha iniciado su formación profesional, en porcentajes semejantes, en un PE del ámbito económico administrativo y un poco más de la mitad estudia un PE del ámbito de las ingenierías.

A su vez, la mayoría de los alumnos vive con sus padres y tiene entre 1 y 3 hermanos, quienes tienen como actividad principal estudiar. Un mayor porcentaje de los estudiantes se dedican únicamente a estudiar, únicamente un porcentaje menor de estudiantes trabaja de 5 a 10 horas semanales. 
No obstante, lo anterior, casi el total de los alumnos dependen de sus padres, quienes tienen ingresos mensuales cercanos a los siete mil pesos, como resultado de ocuparse como empleados. Asimismo, los alumnos son hijos de madres de familia cuya ocupación preponderante son las labores del hogar. Son alumnos, que en su mayoría son hijos de familias que habitan una vivienda propia. Cabe señalar que si bien los alumnos tienen como rol principal el de estudiante hay un porcentaje menor que combina este rol con el de padre o madre de familia.

Se encuentra un perfil de alumno sin problemas de salud física como tampoco existen problemas relacionados con alguna discapacidad, no obstante, en la población estudiantil se tiene problemas de la vista en un porcentaje considerable. A su vez, respecto del estado psicológico del alumnado, se observan problemas de ansiedad, depresión y de aprendizaje, aunque con muy bajos puntajes. Asimismo, se trata de un perfil con bajo nivel de adicciones, con excepción del consumo de alcohol, que tiene presencia en la población estudiantil.

El alumno de la UTNA proviene principalmente de los CECYTE y en la mayoría de los casos el PE al cual ingresaron representa para ellos su primera opción, sin embargo, la mayoría de ellos egresó con un promedio académico que va del 7.1 a 8.5. Por otra parte, se trata de un perfil de estudiante que cuenta con una computadora como herramienta para la realización de sus actividades escolares. A su vez, poco más de la mitad de la población manifestó utilizar los textos en su actividad académica.

\section{Referencias}

CGUTyP (2017). Modelo Nacional de Tutorías. Universidad Tecnológica del Norte de Aguascalientes. (2019). Sistema de Gestión de la Calidad. Rincón de Romos: UTNA. 